\title{
Reliability Conditions in Quadrature Algorithms
}

\author{
Gh. Adam ${ }^{\mathrm{a}, \mathrm{b}, 1}$ S. Adam ${ }^{\mathrm{a}, \mathrm{b}, 2}$ N.M. Plakida ${ }^{\mathrm{a}, 3}$ \\ ${ }^{a}$ Bogolubov Laboratory of Theoretical Physics, Joint Institute for Nuclear \\ Research, 141980 Dubna, Russia \\ ${ }^{\mathrm{b}}$ Department of Theoretical Physics, Institute of Physics and Nuclear Engineering, \\ P.O. Box MG-6, 76900 Bucharest-Măgurele, Romania
}

\begin{abstract}
The detection of insufficiently resolved or ill-conditioned integrand structures is critical for the reliability assessment of the quadrature rule outputs. We discuss a method of analysis of the profile of the integrand at the quadrature knots which allows inferences approaching the theoretical $100 \%$ rate of success, under error estimate sharpening. The proposed procedure is of the highest interest for the solution of parametric integrals arising in complex physical models.
\end{abstract}

PACS: $02.60 . \mathrm{Jh}$, 02.60.Pn, 02.30.Mv

Key words: Numerical integration; Reliability; Interpolatory quadrature; Gauss-Kronrod quadrature; Discretization errors; Oscillatory functions.

\section{Introduction}

A large number of physical models currently under study are characterized by two combined features. First, the observables are obtained as integrals which cannot be solved analytically. Second, the models describe physical systems involving one or more specific parameters the variation of which results in critical modification of the system behaviour. As a consequence, deep understanding of the predictions of the models needs the exploration of the values of the observables over a large range of the variable parameters.

1 Corresponding author; e-mail: adamg@theory.nipne.ro

2 e-mail: adams@theory.nipne.ro

3 e-mail: plakida@thsun1.jinr.ru

Preprint submitted to Elsevier Preprint

19 November 2018 
As usual, to solve the occurring parametric integrals, recourse is made to existing library codes of automatic adaptive quadrature which may fail badly without providing any hint about such possibilities. We are directly aware of three such frustrating experiences. The first one concerns the two-band singlet-hole Hubbard model of cuprate superconductors [1]-[3], which involves integrals over ranges of the first Brillouin zone. The variation of the parameter of the model (the hole or electron doping in the high- $T_{c}$ superconductor) results in substantial modification of the behavior of the involved functions over the Brillouin zone. The exploration of the predictions of the physical model with the doping is fundamental for the validation of the proposed mechanism as responsible for the superconducting pairing in cuprates. However, the reliability of the outputs was found to be exceedingly low to allow sound inferences based on the bare numerical outputs. A similar problem arises in the alternative $\mathrm{U}(1) \times \mathrm{SU}(2)$ gauge theory model of underdoped cuprate superconductors [4]. The meaningful physical solution derived under simplifying assumptions in [5] could not be recovered from outputs generated by the available automatic adaptive quadrature codes. The numerical exploration of a model of nuclear fission [6] could not be achieved by means of library quadrature codes either.

These circumstances come from the fact that the existing algorithms for the numerical integration of real valued functions (see, e.g., [7] for details on the available algorithms and a recent review of numerical quadrature) are tailored for specific classes of integrands, with limited possibilities to solve simultaneously families of integrals falling in different classes.

We may therefore assume that a study able to increase the reliability of the automatic adaptive quadrature algorithms for solving parametric integrals in connection with the exploration of physical models is of interest for a great many users. Within the automatic adaptive quadrature, the approximate value $Q$ of a given integral as well as its associated error estimate $E$ are obtained as sums of local couples $\{q, e\}$ of estimates over subranges.

The general picture offered by the numerical evidence on the solution of parametric integrals points towards the existence of a limited range of parameter values where the local quadrature sum $q$ provides accurate solution of the integral of interest, whereas for other parameter values the quadrature sum $q$ is inaccurate. Over the range of accurate $q$ outputs, the existing quadrature error estimators provide outputs $e$ which, in most cases, grossly overestimate the actual quadrature error, whence the need of supplementary range subdivisions and over computing to meet the input precision requests. However, over the range of inaccurate $q$ outputs, the heuristics implemented in the local error estimators may result in spurious outputs quoted as reliable, hence the impossibility to detect such cases by means of the existing library codes.

In the present paper we discuss a generalization of the approach proposed 
in [8] intended to reconcile these two contradictory aspects. The cornerstone of such an analysis is the derivation of reliability criteria for the validation of the local error estimate $e$ associated to a local quadrature sum $q$ based on the study of the profile of the integrand at the set of quadrature knots entering the expression of $q$.

The basic idea is that an unreliable estimate of $e$ might originate either in the insufficient resolution of the integrand profile, or in the presence of difficult isolated points (integrable singularities, turning points, jumps) which result in slow convergence. The occurrence of each kind of difficulty can be evidenced by means of specific consistency criteria asking for the fulfillment of requirements following from quite general considerations: the very definition of the Riemann integral, the fundamental properties of the basis polynomials which span the approximating linear space where the interpolatory polynomial of the quadrature rule is defined, the properties of the continuous functions at or near their extremal points, and the smoothness properties of the continuous functions inside their monotonicity subranges.

If the integrand is well-conditioned but its profile is insufficiently resolved at the current set of quadrature knots, repeated subdivision of the integration range eventually results in the fulfillment of all the reliability constraints. A genuine difficult integrand point, however, recurs under repeated subrange subdivisions. Therefore, repeated analysis of the integrand profile under subrange subdivision ultimately results in the diagnostics stability under iteration. This is the point where the general control routine of an automatic quadrature rule can take safe decisions concerning the best way to continue the solution refinement or to decide that the integral was solved within the input accuracy specifications.

The paper starts with basic definitions and notations (section 2). In section 3, the main features of the validation procedure of a computed local couple $\{q, e\}$ are discussed. Criteria for the identification of ill-conditioning features within an integrand profile are summarized in section 4 . Their practical importance is assessed in the section 5 based on numerical evidence obtained from the solution of case study integrals by Gauss-Kronrod 10-21 quadrature rules [9] with improved error estimate [8]. Concluding comments are given in section 6 .

\section{Definitions and notations}

Let $I$ denote the actual value of the integral to be solved numerically,

$$
I \equiv I[f]=\int_{a}^{b} g(x) f(x) d x,-\infty<a<b<\infty .
$$


Here, the weight function $g(x)$ is an analytically integrable function which absorbs a difficult part of the integrand (e.g., an oscillatory or a singular factor). In the absence of such factors, $g(x)=1$. The integrand function $f(x)$ is assumed to be continuous almost everywhere on $[a, b]$, such that (1) exists and is finite.

A local quadrature rule produces as solution of (1) a couple $\{q, e\}$, where the quadrature sum $q$ yields an approximate value of the integral $I$, while the local error estimate $e>0$ provides information on the accuracy of $q$. If $e>\left|e_{Q}\right|$, where

$$
e_{Q}=I-q
$$

is the actual error associated to $q$, then the couple $\{q, e\}$ is reliable, otherwise it is unreliable and the numerical solution fails.

A $(2 n+1)$-knot interpolatory quadrature sum $q_{2 n}$ is obtained as the analytical solution of the integral (1) with the integrand $f(x)$ replaced by an interpolatory polynomial of the $2 n$-th degree,

$$
P_{2 n}(x)=\sum_{k=0}^{2 n} \alpha_{k} p_{k}(x),
$$

where $\left\{p_{k}(x)\right\}$ is the set of polynomials of degree at most $2 n$ spanning the approximating space of $P_{2 n}(x)$. The coefficients $\alpha_{k}$ are obtained from the set of conditions of interpolation

$$
P_{2 n}\left(x_{i}\right)=f\left(x_{i}\right),
$$

at a set of $2 n+1$ abscissas (called quadrature knots) inside $[a, b]$,

$$
a \leq x_{0}<x_{1}<\cdots<x_{2 n} \leq b
$$

In the particular case of the symmetric $(2 n+1)$-knot quadrature sums, the interpolation abscissas inside $[a, b]$ are given by

$$
x_{i}=c+h y_{i} ; c=(b+a) / 2 ; h=(b-a) / 2 ; i=-n,-n+1, \cdots, n,
$$

where the reduced quadrature knots $y_{i}$ are defined on $[-1,1]$, such that $0=$ $y_{0}<y_{1}<y_{2}<\cdots<y_{n} \leq 1$, while $y_{-i}=-y_{i}, i=1, \cdots, n$. 
The local quadrature sum $q_{2 n}$ is then expressed as a linear combination of the integrand values at the quadrature knots,

$$
q_{2 n} \equiv Q_{2 n}[f]=\sum_{i=-n}^{n} w_{i} f\left(x_{i}\right),
$$

with the quadrature weights showing the symmetry property $w_{-i}=w_{i}$.

The information provided by the $2 n+1$ integrand values at the quadrature knots, $\left\{f\left(x_{i}\right) \mid i=-n, \cdots, n\right\}$, is insufficient for the derivation of an expression for the error estimate $e_{2 n}$ associated to $q_{2 n}$.

Kronrod [10] derived an error estimate, called in what follows genuine GaussKronrod (ggk) error estimate, from an upper bound of

$$
e_{g g k}=\left|q_{2 n}-q_{n}\right|
$$

where $q_{2 n}$ is the quadrature sum (7), while $q_{n}$ is a lower degree quadrature sum derived over the subset of (6),

$$
x_{-n+\gamma}<x_{-n+\gamma+2}<\cdots<x_{n-\gamma-2}<x_{n-\gamma} .
$$

Here, $\gamma=1$ for an open quadrature sum (typically, the Gauss-Kronrod (GK) quadrature where the spanning basis $\left\{p_{k}(x)\right\}$ in (4) is given by Legendre polynomials and their orthogonal Kronrod extensions), while $\gamma=0$ for a closed quadrature sum (typically, the Clenshaw-Curtis (CC) quadrature where the spanning basis $\left\{p_{k}(x)\right\}$ in (4) is given by Chebyshev polynomials).

In what follows, the set of quadrature knots (6) is referred to as the fine discretization of the integration domain $[a, b]$, while the sparser set of quadrature knots (9) as the coarse discretization of $[a, b]$. The integrand values at these knots define its fine and coarse samplings respectively.

In the QUADPACK package [9], which has been incorporated in most major program libraries, while a ggk error estimate was implemented for the CC quadrature, the GK error estimate was reformulated as follows. Let $\bar{f}$ denote the computed value of the average of $f(x)$ over $[a, b]$ at the knots $(6)$,

$$
\bar{f}=q_{2 n} /(b-a)
$$

and let $\Delta=Q_{2 n}[|f-\bar{f}|]$ denote the computed value of $\int_{a}^{b}|f(x)-\bar{f}| d x$, which measures the area covered by the deviations of $f(x)$ around $\bar{f}$. 
The local QUADPACK error estimate (qdp) is then given by

$$
e_{q d p}=\Delta \times \min \left\{\left(200 e_{g g k} / \Delta\right)^{3 / 2}, 1\right\} .
$$

The values (8) and (11) are taken for error estimates provided they do not fall below the attainable accuracy limit imposed by the relative machine precision. The latter threshold is defined as the product

$$
e_{\text {roff }}=\tau_{0} \epsilon_{0} Q_{2 n}[|f|] .
$$

Here $\tau_{0}$ is an empirical multiplicative factor (following QUADPACK, we have chosen $\left.\tau_{0}=50\right)$ and $\epsilon_{0}$ denotes the relative machine accuracy.

For the case study integrals considered below, the value $I$ of $(1)$ is computed from the existing analytical expressions, such that the exact error $e_{Q}(2)$ of the quadrature sum $q_{2 n}$ can be defined.

In the graphical representation of the quadrature errors, the moduli of the relative errors (simply called relative errors in the sequel) are useful,

$$
\varepsilon_{\alpha}=\left|e_{\alpha} / I\right|, \quad \alpha \in\{2 n, Q\} .
$$

The derivation of the local error estimates within a subroutine which implements a quadrature rule uses information inferred from the estimated relative errors,

$$
\rho_{\alpha}=\left|e_{\alpha} / q_{2 n}\right|, \quad \alpha \in\{g g k, q d p, 2 n\} .
$$

\section{Stability of the diagnostics under subrange subdivision}

Using (8), (11) and (12), we get the local error estimate [8]

$$
e_{2 n}=\max \left[e_{r o f f}, \min \left(e_{g g k}, e_{q d p}\right)\right],
$$

the reliability of which is almost always subject to doubt, except for the case when the lower degree quadrature sum $q_{n}$ is sufficiently accurate such that the accuracy of $q_{2 n}$ itself reaches nine to ten significant figures at least. Such a condition can be confidently assumed to hold provided

$$
e_{2 n}>e_{t h r}, \quad e_{t h r}=2^{-18} \simeq 0.38 \times 10^{-5} .
$$


This empirically proposed threshold value is about two decimal figures more conservative than the smallest values of the unreliable computed error estimates over the evidence discussed in Sec. 5.

If the opposite of (16) occurs, then a validation procedure is to be used to assess the reliability of the local couple $\left\{q_{2 n}, e_{2 n}\right\}$. Thus, a self-validating quadrature rule returns, besides the numerical output for $e_{2 n}$, a flag having the value zero in case of assumed reliable outputs and non-zero value if the output is not validated.

The validation procedure proposed in this paper is based on the study of the information contained in the profile of the integrand $f(x)$ over $[a, b]$, defined as the set of integrand values at the quadrature knots (5), completed with the endpoint values $f(a)$ and $f(b)$ in the case of open quadrature sums. Since the operation of subrange subdivision within automatic adaptive quadrature always involves inner abscissas at existing quadrature knots, the only price to be paid for the inclusion of the endpoint values in the integrand profile is the direct access of the general control routine to such data. This goal is achieved provided the generation of the integrand sampling at the quadrature knots (5) is done within a subroutine which is distinct from that implementing the quadrature rule and is directly subordinated to the general control routine.

The study of the integrand profile starts with the definition of its monotonicity subranges, $\left[x_{i_{j-1}}, x_{i_{j}}\right]$, over $[a, b]$, where

$$
a=x_{i_{0}}<x_{i_{1}}<x_{i_{2}}<\cdots<x_{i_{m}}<x_{i_{m+1}}=b,
$$

denote the abscissas of the extremal points of $f(x)$ within the sampling.

In terms of the answer concerning the number of monotonicity subranges, several specific reliability criteria are checked and the number $\lambda$ of the infringements of these criteria is counted. There are three critical values of the pointer $\lambda$ in terms of which a decision is taken:

- $\lambda=0$ : probably the investigated integrand profile comes from a wellconditioned integrand, hence the output $q_{2 n}$, Eq. (7) is reliable, while the quadrature error estimate (15) is overestimated.

- $\lambda=1$ or $\lambda=2$ : there is a high probability that a difficult isolated point is present which implies slow convergence of the quadrature sums.

- $\lambda \geq 3$ : the insufficient resolution of the integrand profile at the involved quadrature knots is manifest. The output is useless and further subrange subdivisions are compulsory.

The existence and finiteness of the Riemann integral (1) guarantees that, after a finite number of subrange subdivisions, the discretization process will reach 
a stable profile configuration the refinement of which will result in unessential modifications only.

Under the occurrence of isolated difficult points of the integrand, the discretization process will resolve the profile over the well-conditioned subranges within a finite number of subrange subdivisions, and then it will mainly create a dense mesh around the difficult points. In this case, the automatic control subroutine will safely decide upon the activation of a specific convergence acceleration algorithm, such that a reliable numerical solution will be available in the end.

The achievement of the stability of the diagnostics concerning the conditioning properties of the integrand profiles over subranges, got after a finite number of subrange subdivisions, is the fundamental feature which secures the efficiency of the procedure proposed in this investigation.

The occurrence of consistent with each other reliability diagnostics over the current integration range and its subranges obtained by subrange subdivision enables the general control routine to take safe decisions concerning the activation of the implemented alternative algorithms.

\section{Well-conditioned integrand profiles}

The consistency requirements satisfied by a well-conditioned integrand profile are formulated mostly locally and they follow from quite general considerations which are discussed in the next subsections.

Any infringement of the consistency criteria derived below is to be added to the value of the ill-conditioning pointer $\lambda$.

\subsection{Insensitivity of the integral sums to discretization details}

The standard definition of the integral sums in a Riemann integral assumes the fulfillment of the following two features:

(i) The norm of the discretization step defined over the integration domain tends to zero.

(ii) The integral sum is insensitive to the the addition or removal of a single discretization abscissa within the defined partition.

In the quadrature algorithms, the norm of the discretization (6) is far from being close to zero. The quadrature knots are not distributed evenly either. 
For the GK and CC quadrature rules mentioned above, the fundamental range $[-1,1]$ consists of a sparser knot region centered around the origin and two denser knot regions located toward the range ends. The number of abscissas entering the integrand profile associated to a $(2 n+1)$-knot open quadrature rule equals $2 n+3$, while the corresponding number for a closed quadrature rule is $2 n+1$. Therefore, for both kinds of quadrature rules, a particular inner reduced knot $y_{i}$ lies in the dense knot region provided the lengths of its two adjacent subranges are smaller than the threshold quantity for a uniform distribution, $d_{a v}=2 /(2 n+3)$.

An immediate consequence of the feature (i) is the property that the denser discretization regions of a smooth integrand $f(x)$ secure better accuracy of their contributions to the quadrature sums than the sparser ones. We reformulate this observation as follows: the generation of the fine discretization (6) from the coarse discretization (9) is expected to result in non-essential modifications of the profile of $f(x)$ over the regions of dense knot discretization.

To characterize the extent to which a profile is modified by the addition of new knots inside the region of dense knot discretization, let us consider that $x_{0}$ is such a knot. If $x_{0}$ belongs to the set of extremal points (17) such that the integrand value $f\left(x_{0}\right)$ is isolated from the integrand values $f\left(x_{-1}\right)$ and $f\left(x_{1}\right)$ at the nearest neighbours $x_{-1}$ and $x_{1}$ by the median line $f=\bar{f}$, Eq. (10), then the knot $x_{0}$ is said to be sensitive. If both quantities $f\left(x_{-1}\right)$ and $f\left(x_{1}\right)$ stay on the same side with $f\left(x_{0}\right)$ with respect to the median line $f=\bar{f}$, then the knot $x_{0}$ is said to be regular. If the median line $f=\bar{f}$ separates $f\left(x_{0}\right)$ from only one of the values $f\left(x_{-1}\right)$ or $f\left(x_{1}\right)$, then the knot $x_{0}$ is said to be gray.

We are now ready to formulate the first practical reliability criterion:

(I) Non-sensitivity of the extremal points:

The addition of supplementary quadrature knots to the coarse partition (9) to reach the fine partition (6) does not result in supplementary gray or sensitive extrema of the profile of a well-conditioned integrand over the regions of dense knot discretization.

\subsection{Features which stem from the basis polynomials}

Since the equations (6) perform the mapping of the original interval $[a, b]$ onto the reduced interval $[-1,1]$ over which the orthogonal polynomials are usually defined, in this subsection we refer to this reduced interval and use the notation $p_{k}(y)$ for the basis polynomials. All the properties discussed below hold true over arbitrary interval lengths, hence reference to the expression (3) of the interpolatory polynomial spanned by the basis orthogonal polynomials does not give rise to any confusion. 
The existence and uniqueness of the interpolatory polynomial (3) is secured provided the set of basis polynomials spanning (3) define a Chebyshev system over $[a, b]$. Therefrom the following properties hold true:

(iii) $p_{0}(y)=$ const.

(iv) The set of the successive extremal values of a polynomial $p_{k}(y)$ of degree $k>1$ defines an alternating sequence over $[-1,1]$.

(v) The zeros of the polynomials $p_{k}(y)$ and $p_{k+1}(y)$ are interlaced inside the open range $(-1,1)$.

The average value $\bar{f}$, Eq. (10), of the integrand $f(x)$, which defines its zeroth order moment over the sampling (5) and is related to the coefficient of $p_{0}(y)$ within a basis set of orthogonal polynomials, serves as reference value with respect to which the oscillations of the integrand profile are counted. The intersections of the integrand profile with the line $f=\bar{f}$ define the zeros of the integrand profile.

The alternation property (iv) results in the important consequence that the deviations of the successive extremal values of a well-conditioned integrand profile from $\bar{f}$ define an alternating sequence with comparable amplitudes at the adjacent extremal knots (17). This property can be detailed for practical purposes into two well-conditioning alternation criteria:

(IIa) Type-1 alternation criterion:

- Each inner monotonicity subrange of a well-conditioned integrand profile intersects the line $f=\bar{f}$.

- The two end point monotonicity subranges do not diverge from $f=\bar{f}$.

(IIb) Type-2 alternation criterion:

Each inner gray extremal point which satisfies the type-1 alternation criterion is to stay sufficiently far from the line $f=\bar{f}$.

The test for the occurrence of an infringement of the type- 1 alternation criterion is obvious. As it concerns the the latter criterion, two infringements are to be simultaneously tested:

- The distance from $f\left(x_{0}\right)$ to $\bar{f}$ is to be smaller than those of its nearest neighbouring extrema.

- Let $a_{0}, a_{l}$ and $a_{r}$ denote the areas surrounded by $f=\bar{f}$ and the integrand profile around $x_{0}$ and its nearest neighbours in the set (17). Then

$$
\left|a_{0}\right|<t_{1}\left|a_{l}+a_{r}\right|, \quad t_{1}=10,
$$

where the value of $t_{1}$ was chosen such as to point to a discrepancy exceeding an order of magnitude. The computation of the three local areas is done by compound trapeze rule which is robust and sufficiently accurate for the involved comparison. 
Corroboration of the interlacing property (v) with the non-sensitivity criterion (I) results in a criterion for the distribution of the zeros of the integrand profile:

(III) Non-sensitivity of the zeros:

Over the dense knot regions, the numbers of zeros of the fine and coarse profiles of a well-conditioned integrand are the same.

\subsection{Integrand variations around its isolated extremal points}

The lateral first order derivatives of a smooth first order differentiable function vanish at an extremal point, while the curvature of a second order differentiable function (which is given by the second order derivative) keeps constant sign over a nonvanishing neighbourhood of the extremum.

Within the discrete mesh defined by the quadrature knots, inquiries about these properties can be made only at integrand profile approximations of isolated extremal points of the integrand. If $x_{0}$ is such a point, then a sufficiently large neighbourhood $\left\{\xi_{l}, \xi_{r}\right\}$ around $x_{0}$ can be defined within which the evaluation of the quantities of interest is expected to be weakly influenced by the presence of neighbouring extrema.

Let us assume that an isolated extremal point of a well-conditioned integrand was identified within a sufficiently well resolved integrand profile. The following consistency criteria establish well-conditioned behaviours of the data:

(IV) First lateral derivative criterion:

The approximation of the lateral first order derivatives at an isolated extremum of the profile using fine sampling data is closer to zero as compared to the value estimated from data defined over a coarse sampling with respect to the extremum location.

(V) Curvature sign constancy criterion:

The sign of the second order derivative computed from fine sampling data centered at the extremum is the same as that of the value estimated from data involving the reference extremum as a lateral point to the left/right.

We shall illustrate the quantitative implementation of these criteria for a reference extremum $x_{0}$ which is said to be isolated to the right. That is, the neighbourhood $\left\{\xi_{l}, \xi_{r}\right\}$ contains inside it the set of abscissas $\left\{x_{-1}, x_{0}, x_{1}, x_{2}\right\}$ at which the integrand function takes respectively the values $\left\{f_{-1}, f_{0}, f_{1}, f_{2}\right\}$.

To estimate the approximation of the first order right lateral derivative, we define the interpolatory polynomial of the third degree which fits these four 
data. This yields the following result:

$$
f_{r, f i n e}^{\prime}\left(x_{0}\right)=d_{1,0}^{(1)}-\frac{h_{1,0}}{h_{2,-1}}\left[h_{0,-1} d_{2,1}^{(2)}+h_{2,0} d_{1,-1}^{(2)}\right] .
$$

Here, $h_{i, j}=x_{i}-x_{j}, d_{i, j}^{(1)}=\left(f_{i}-f_{j}\right) / h_{i, j}$ denote the first order divided differences at $x_{i}$ and $x_{j}$, while $d_{2,1}^{(2)}=\left(d_{2,0}^{(1)}-d_{1,0}^{(1)}\right) / h_{2,1}$ and $d_{1,-1}^{(2)}=\left(d_{1,0}^{(1)}-d_{0,-1}^{(1)}\right) / h_{1,-1}$ denote specific second order divided differences.

On the other hand, the coarse sampling around $x_{0}$ yields:

$$
f_{r, \text { coarse }}^{\prime}\left(x_{0}\right)=d_{2,0}^{(1)}
$$

The criterion (IV) then simply states that the approximations (19) and (20) should satisfy $\left|f_{r, \text { fine }}^{\prime}\left(x_{0}\right)\right|<\left|f_{r, \text { coarse }}^{\prime}\left(x_{0}\right)\right|$.

Over the same set of data, the criterion (V) requirement of constancy of the sign of the second order derivative results in the condition

$$
\left(d_{2,0}^{(1)}-d_{1,0}^{(1)}\right)\left(d_{1,0}^{(1)}-d_{0,-1}^{(1)}\right)>0 .
$$

For the extremal point $x_{0}$ isolated to the left, similar conditions are derived from the data set $\left\{f_{-2}, f_{-1}, f_{0}, f_{1}\right\}$ obtained at the abscissas $\left\{x_{-2}, x_{-1}, x_{0}, x_{1}\right\}$.

\subsection{Well-conditioning inside monotonicity subranges}

Inside any monotonicity subrange of a smooth first order differentiable function $f(x)$, the first order derivative varies smoothly from point to point.

Within numerical quadrature, the fulfillment of this property for an integrand sampling can be checked by making use of first order divided differences. If the integrand profile is monotonic over $[a, b]$, or monotonicity subranges can be defined which extend over three successive knots at least, then a smoothly varying profile will by characterized by the absence of jumps:

(VI) Absence of jumps inside monotonicity subranges:

Inside a monotonicity range, the ratio of two successive first order divided differences cannot exceed a relative smoothness threshold.

If one of the knots involved in the divided differences is an extremal point, then this smoothness condition is to be checked only one-directionally, skipping the case of vanishingly small divided difference at the extremal point. 
For knots far from inflection points, a threshold value $t_{j m p}=10$, corresponding to the agreement of the successive divided differences within an order of magnitude, is appropriate. In the neighbourhood of inflection points characterized by a maximum of the first order derivative, this value is to be halved to detect ill-conditioned behaviour, while in the neighbourhood of inflection points characterized by a minimum of the first order derivative, five times larger threshold value is appropriate.

\section{$5 \quad$ Numerical results}

The significance of the conditioning criteria discussed in the previous section is intuitive and straightforward. In addition to the case specified by the condition (16), a second case when the reliability analysis can be skipped is that of a monotonic profile characterized by an error estimate

$$
e_{2 n}>0.5\left|q_{2 n}\right|
$$

Then the computed quadrature sum is highly inaccurate, such that an error flag can be directly assigned.

The diagnostics of the reliability criteria (IIb), (IV), (V), and (VI) depend on specific adjustable parameters. If the quantitative thresholds entering these criteria are decreased, the diagnostics becomes less permissive, with the consequence that the reliability range shrinks and the number of wrong diagnostics is decreased. The opposite occurs under the increase of the quantitative thresholds. The numerical data reported in this section show that, when corroborated with the requirement of the stability of the diagnostics formulated in Sec. 3, the formulation of the reliability criteria in Sec. 4 is able to eliminate practically all the spurious outputs occurring in an automatic adaptive quadrature algorithm.

To illustrate the present analysis, a comparison is done of three codes using Gauss-Kronrod 10-21 (GK 10-21) quadrature rules: $(\alpha)$ the QUADPACK code [9], $(\beta)$ the self-validating code of $[8]$, and $(\gamma)$ the code using the present reliability analysis.

Each code solved the parametric families of elementary integrals considered in ref. [8]. 
The first is the family of integrals over $[0,1]$ of the terms of the fundamental power series, $x^{n}$,

$$
\int_{0}^{1} x^{n} d x=\frac{1}{n+1}, \quad n=0,1, \cdots, 1023 .
$$

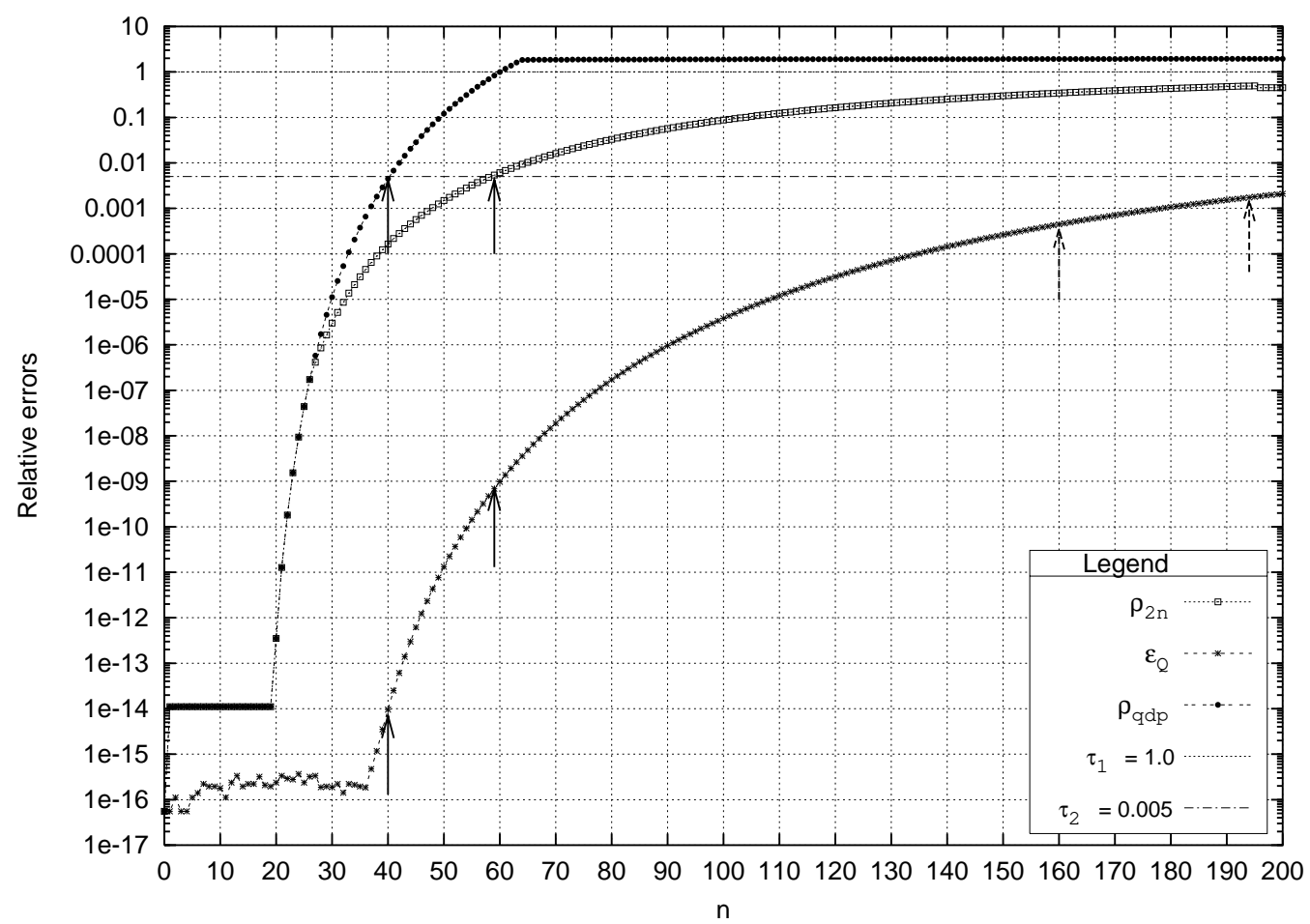

Fig. 1. Relative errors $\rho_{q d p}$ and $\rho_{2 n}$, Eq. (14), $\varepsilon_{Q}$, Eq. (13), of the GK 10-21 outputs for the family of integrals (23) at exponents $n \leq 200$. The upper leftmost solid line arrow points to the accuracy basin extension established by the QUADPACK code using the error estimate (11). The solid line arrow on the same vertical points to the upper accuracy of the quadrature sum $q_{2 n}$ retained as reliable by the QUADPACK code. The next pair of solid arrows show the result of the analysis done in ref. [8]. The left interrupted line arrow represents the extension of the reliability basin established by the present analysis, while the right one shows the exponent threshold above which the criterion (22) supersedes the need of reliability analysis.

The integrands are monotonic, inflection points are absent over the integration range. Fig. 1 illustrates the behaviour of the error estimates with the power $n$ running over the range $\{0,200\}$. The results obtained for this family of integrals can be summarized as follows:

- The QUADPACK code infers an accuracy basin of the GK 10-21 code extending from $n=0$ to $n_{\max }=40$, with the consequence that all the $q_{2 n}$ outputs showing an actual accuracy lower than 14 decimal digits are thrown away. As shown in [8], this early cut of the accuracy basin does not rule out the possibility of wrong error diagnostics at asymptotically large $n$. 
- The self-validating analysis of ref [8] extends the accuracy basin of GK 10-21 up to $n_{\max }=59$, which corresponds to a correct identification of the outputs $q_{2 n}$ as reliable up to accuracies of roughly nine significant digits. Above $n \geq 60$, all the reliability diagnostics have been correct.

- The present analysis establishes an accuracy basin up to $n_{\max }=160$, which corresponds to outputs $q_{2 n}$ showing at least three significant decimal digits. At exponents $n \geq 195$, the criterion (22) directly establishes the occurrence of unreliable $q_{2 n}$ outputs without making recourse to the reliability analysis.

The second family solves integrals for a same integrand (which simulates a centrifugal potential at large $x$ ) over ranges of variable length,

$$
\int_{0}^{b} \frac{1}{x^{2}+1} d x=\arctan (b), \quad b=n, \quad n=0,1, \cdots, 10000 .
$$

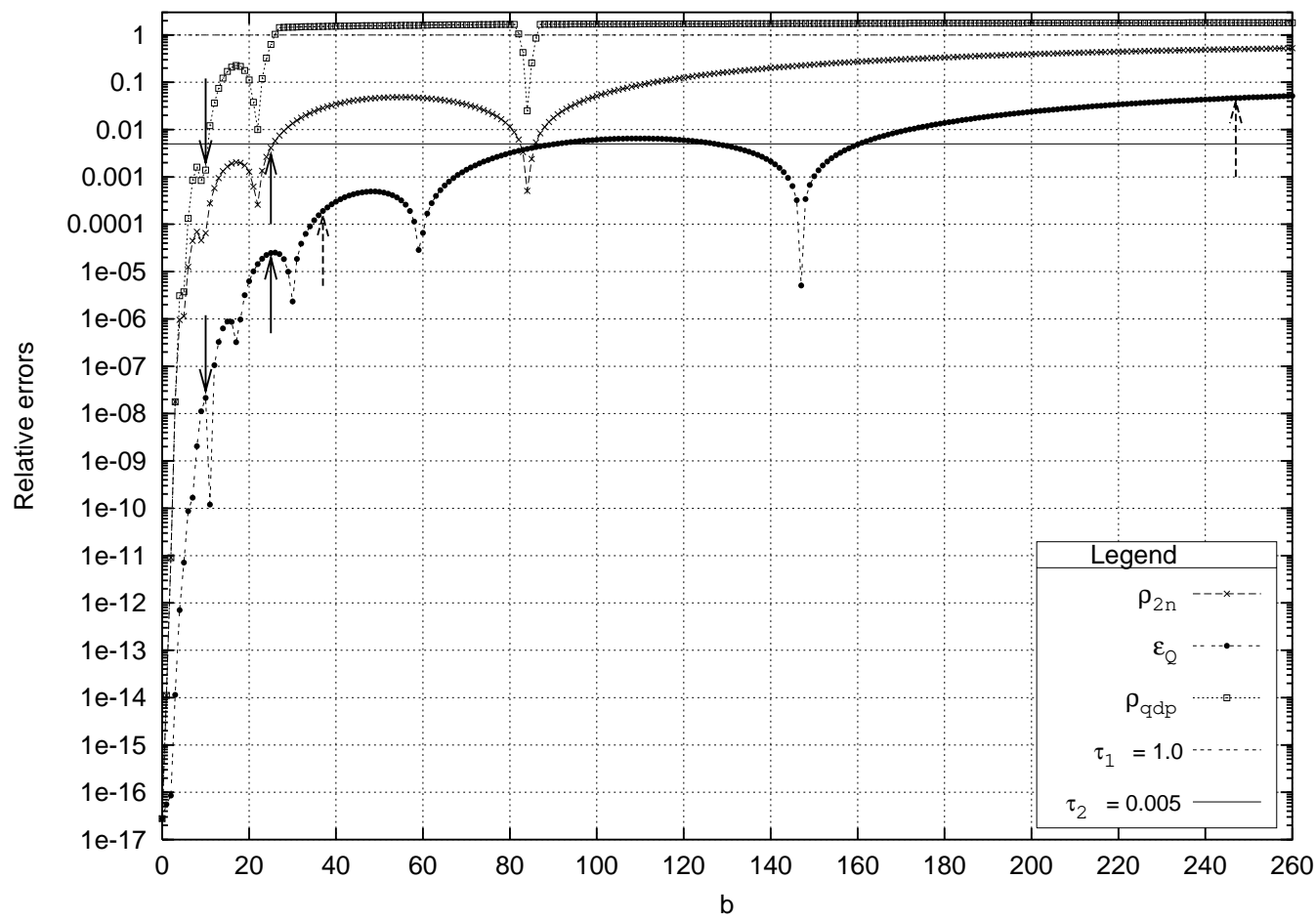

Fig. 2. Same as fig. 1 for the family of integrals (24), at upper integration ranges $b \leq 260$. The arrows bear the same significance.

The integrands are monotonic, an inflection point is present. Fig. 2 illustrates the behaviour of the error estimates with the upper integration range $b=n$ for $n$ running over the range $\{0,260\}$. In this figure the occurrence of cusps in the $\varepsilon_{Q}$ curve points to the existence of fractional integration domain lengths at which the quadrature sum $q_{2 n}$ solves exactly the integral (24), such that the exact error changes sign. The results obtained for this family of integrals can be summarized as follows: 
- The QUADPACK code infers an accuracy basin of the GK 10-21 code extending up to $n_{\max }=10$, with the consequence that all the $q_{2 n}$ outputs showing an actual accuracy lower than seven decimal digits are thrown away. All the QUADPACK reliability diagnostics above $b=n=2460$ have been false.

- The self-validating analysis of ref [8] extends the accuracy basin of GK 10-21 up to $n_{\max }=25$, which corresponds to a correct identification of the outputs $q_{2 n}$ as reliable up to accuracies of about six significant digits. At $n \geq 26$, all the reliability diagnostics have been correct.

- The present analysis establishes an accuracy basin up to $n_{\max }=37$, which corresponds to outputs $q_{2 n}$ showing at least three significant decimal digits. At exponents $n \geq 247$, the criterion (22) directly establishes the occurrence of unreliable $q_{2 n}$ outputs without making recourse to the reliability analysis.

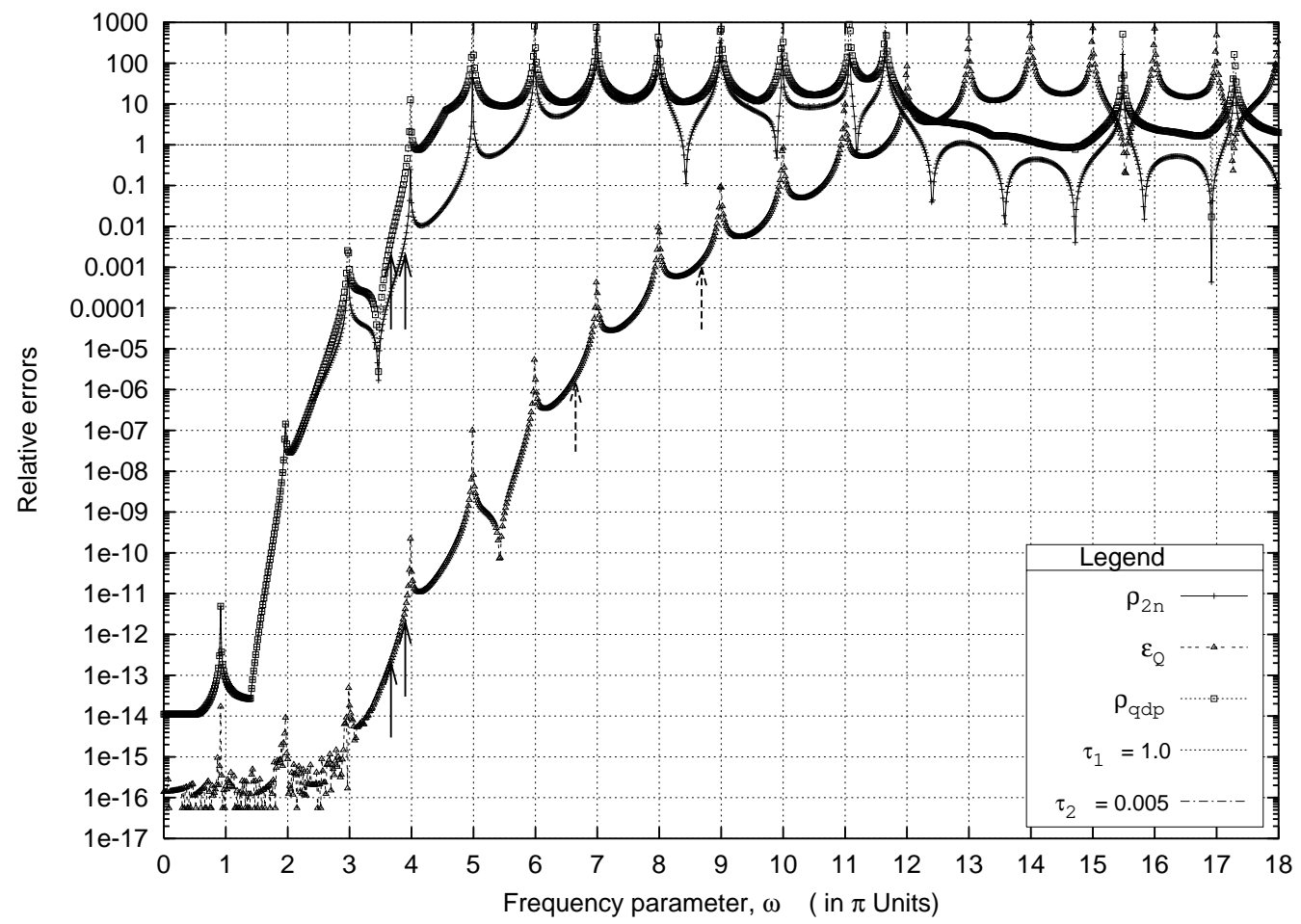

Fig. 3. Outputs of the GK 10-21 quadrature rule for the family of integrals (25) at $p=1$. The significances of the solid line arrows are the same as in fig. 1 . The left interrupted line arrow represents the extension of the reliability basin up to which the present analysis validates all the outputs $q_{2 n}$. Inbetween the two interrupted line arrows, the diagnostics of the present analysis is too conservative in about one third of the solved cases.

Next, we considered two pairs of families of integrals showing nonmonotonic (oscillatory) behaviour, written in algebraically equivalent forms:

$$
\text { (C1) } \int_{-1}^{1} e^{p\left(x-x_{0}\right)} \cos (\omega x) d x=
$$




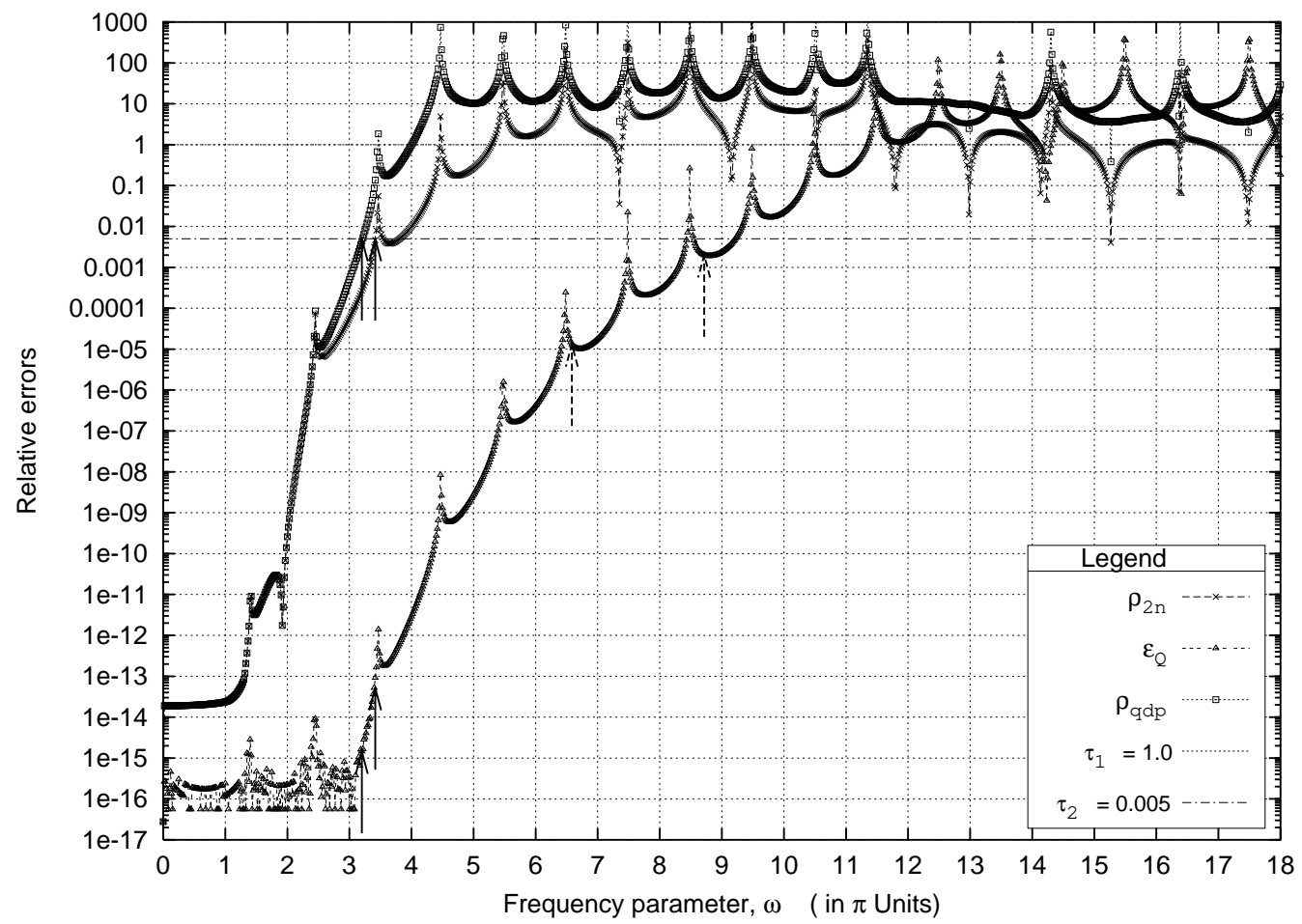

Fig. 4. Same as fig. 3 for the family of integrals (28) at $p=1$.

(C2) $\begin{aligned} & \int_{0}^{1} 2 e^{-p x_{0}} \cosh (p x) \cos (\omega x) d x= \\ = & 2 e^{-p x_{0}}[p \sinh (p) \cos (\omega)+\omega \cosh (p) \sin (\omega)] /\left(\omega^{2}+p^{2}\right)\end{aligned}$

(S1) $\int_{-1}^{1} e^{p\left(x-x_{0}\right)} \sin (\omega x) d x=$

(S2) $\int_{0}^{1} 2 e^{-p x_{0}} \sinh (p x) \sin (\omega x) d x=$

$=2 e^{-p x_{0}}[p \cosh (p) \sin (\omega)-\omega \sinh (p) \cos (\omega)] /\left(\omega^{2}+p^{2}\right)$.

The parameter $\omega$ was chosen to run over the set of values

$$
\omega_{n}=n \pi / 60, \quad n \in\{0,6000\},
$$

while constant values $p=1$ and $x_{0}=-1$ have been chosen on the ground that they are typical for the description of the behaviour of the numerical results.

The analysis of the families of integrals (25-29) shows that the identification of a well-conditioned nonmonotonic integrand profile needs testing the complete 


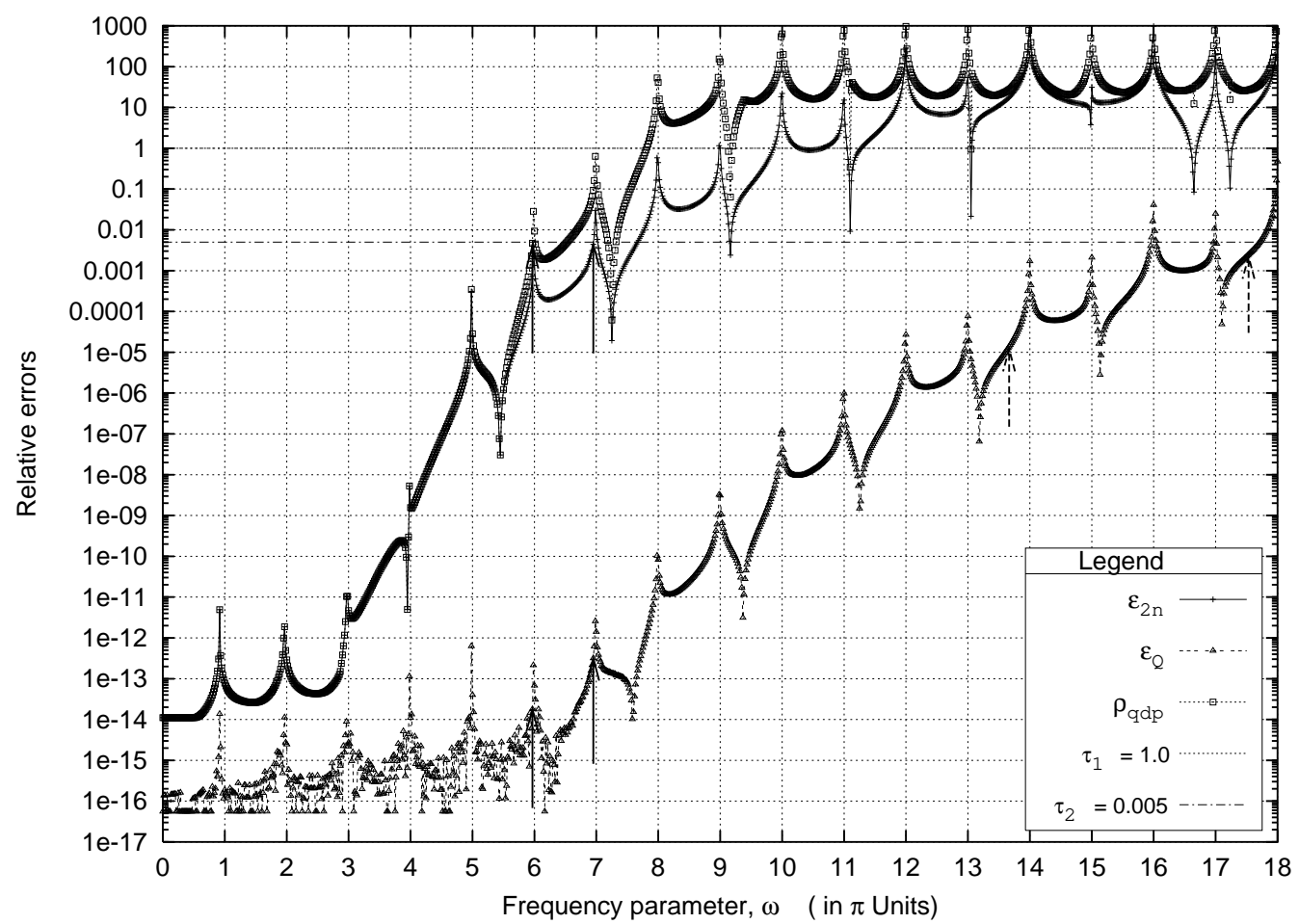

Fig. 5. Same as fig. 3 for the family of integrals (26) at $p=1$.

set of consistency criteria established in Sec. 4. Therefore, the analysis is long. However, it is straightforward and can be easily implemented in a computer program.

Figures 3 to 6 show outputs for the parameter $n$ running over the range $n \in$ $\{0,1080\}$. In these figures, two peculiarities of the $\varepsilon_{Q}$ curves are apparent. Similar to Fig. 2, the occurrence of cusps at minima in the $\varepsilon_{Q}$ curves point to the existence of values of the parameter $\omega$ at which the given integrals are solved exactly by the quadrature sum $q_{2 n}$, such that the exact error changes sign. The sharp maxima noticed in the $\varepsilon_{Q}$ curves occur at $\omega$ values which correspond to entire periods of the oscillatory factors over the integration range, such that important cancellation by subtraction effects occur which result in sensible worsening of the numerical output.

A summary of the results obtained for the families of integrals (25), (26), (28), and (29) is given in Table 1.

The QUADPACK code predicts the narrowest accuracy basins in all the cases. Practically, any computed output $q_{2 n}$ with actual accuracy above the computer roundoff is ruled out as unreliable. At large values of the argument $\omega$ of the trigonometric functions, this code results in an average rate of spurious outputs of about two percent. In figs. 3 and 4 , unreliable estimates of this code are noticed at arguments $\omega>12 \pi$ and $\omega>14 \pi$ respectively. The user is not notified of the wrong diagnostics associated to these outputs at the moment 


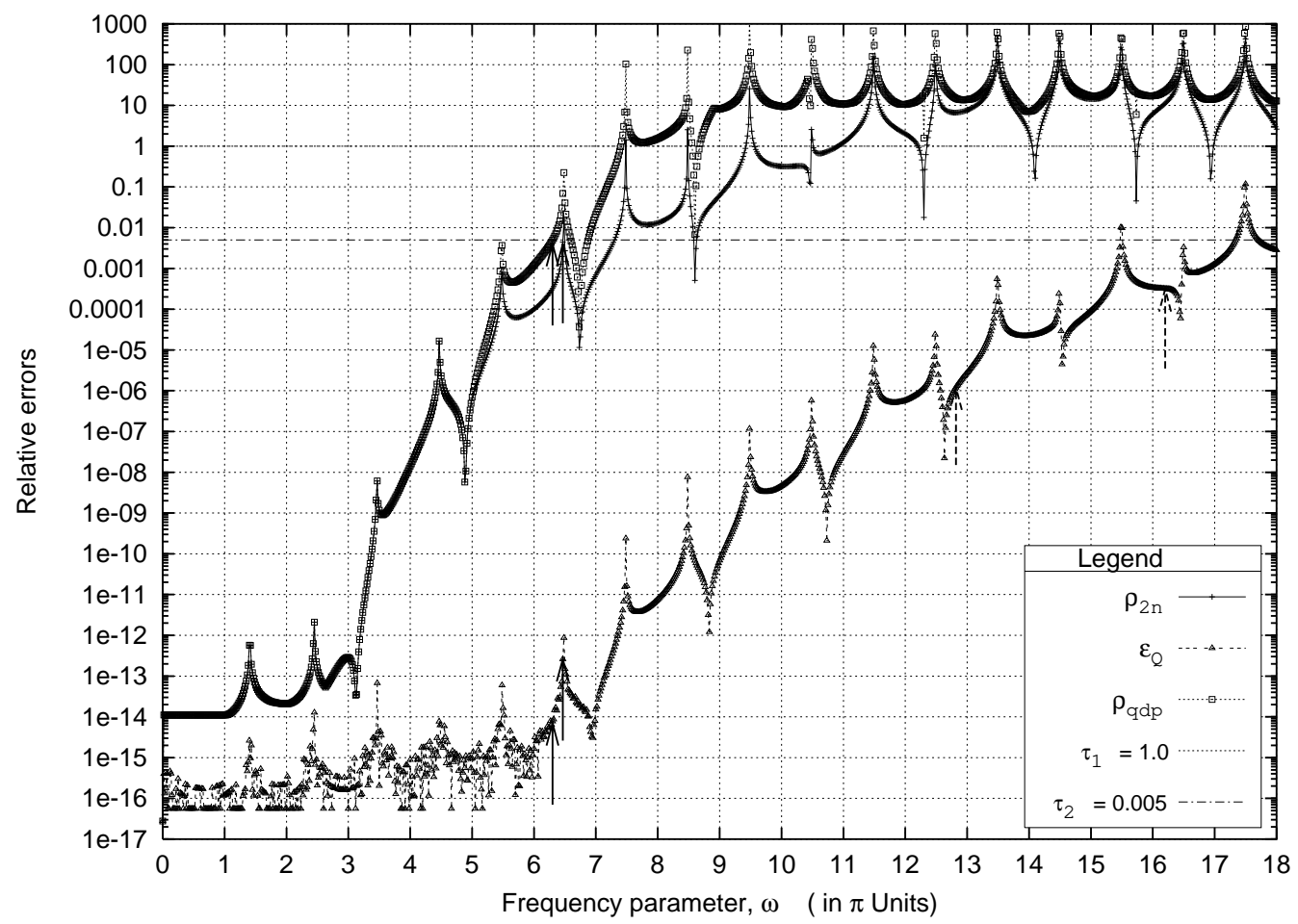

Fig. 6. Same as fig. 3 for the family of integrals (29) at $p=1$.

of solving the integrals of interest. As mentioned in the Introduction, the only way of identifying them is the far end prediction of nonphysical results for the involved observables.

Table 1

Comparison of the stability basins and diagnostics reliability of the three codes

\begin{tabular}{|l|cccc|cccc|}
\hline & \multicolumn{3}{|c|}{$\begin{array}{c}\text { Extension } n_{\max } \\
\text { of the accuracy basins }\end{array}$} & \multicolumn{4}{c|}{$\begin{array}{c}\text { Number of spurious } \\
\text { diagnostics at output }\end{array}$} \\
\hline Integral family & $(\mathbf{C 1})$ & $(\mathbf{S 1})$ & $(\mathbf{C 2})$ & $(\mathbf{S 2})$ & $(\mathbf{C 1})$ & $(\mathbf{S 1})$ & $(\mathbf{C 2})$ & $(\mathbf{S 2})$ \\
\hline QUADPACK & 219 & 192 & 359 & 379 & 254 & 33 & 115 & 61 \\
\hline Ref. [8] & 233 & 202 & 417 & 389 & 0 & 0 & 0 & 0 \\
\hline Present & 399 & 395 & 820 & 769 & 26 & 46 & 41 & 7 \\
& $(521)$ & $(523)$ & $(1052)$ & $(1069)$ & $(0)$ & $(0)$ & $(0)$ & $(0)$ \\
\hline
\end{tabular}

*) For the present analysis, the upper values correspond to the left interrupted line arrows in Figs. 3 to 6 . The values under parentheses correspond to the right interrupted line arrows in the same figures.

${ }^{* *)}$ For the present analysis, the upper values show the number of primary analysis failures. The vanishing values under parentheses show that all the primary analysis failures were corrected under subrange subdivision. 
The self-validating procedure developed in ref. [8] slightly enlarged the extension of the accuracy basin predictions, with no wrong outputs at all.

The present reliability analysis identified substantially larger accuracy basins of the output. All the $q_{2 n}$ outputs showing more than six accurate figures have been correctly identified as reliable. For outputs $q_{2 n}$ showing inbetween six and three accurate figures, the present diagnostic was too conservative for 19 (C1) integrals, 51 (S1), 57 (C2), and 176 (S2) integrals. At values of the argument $\omega$ in large excess to those falling in the accuracy basins, a number of spurious diagnostics was produced by the primary reliability analysis. All the wrong diagnostics occurring at a first run were identified as wrong and corrected under subrange subdivision.
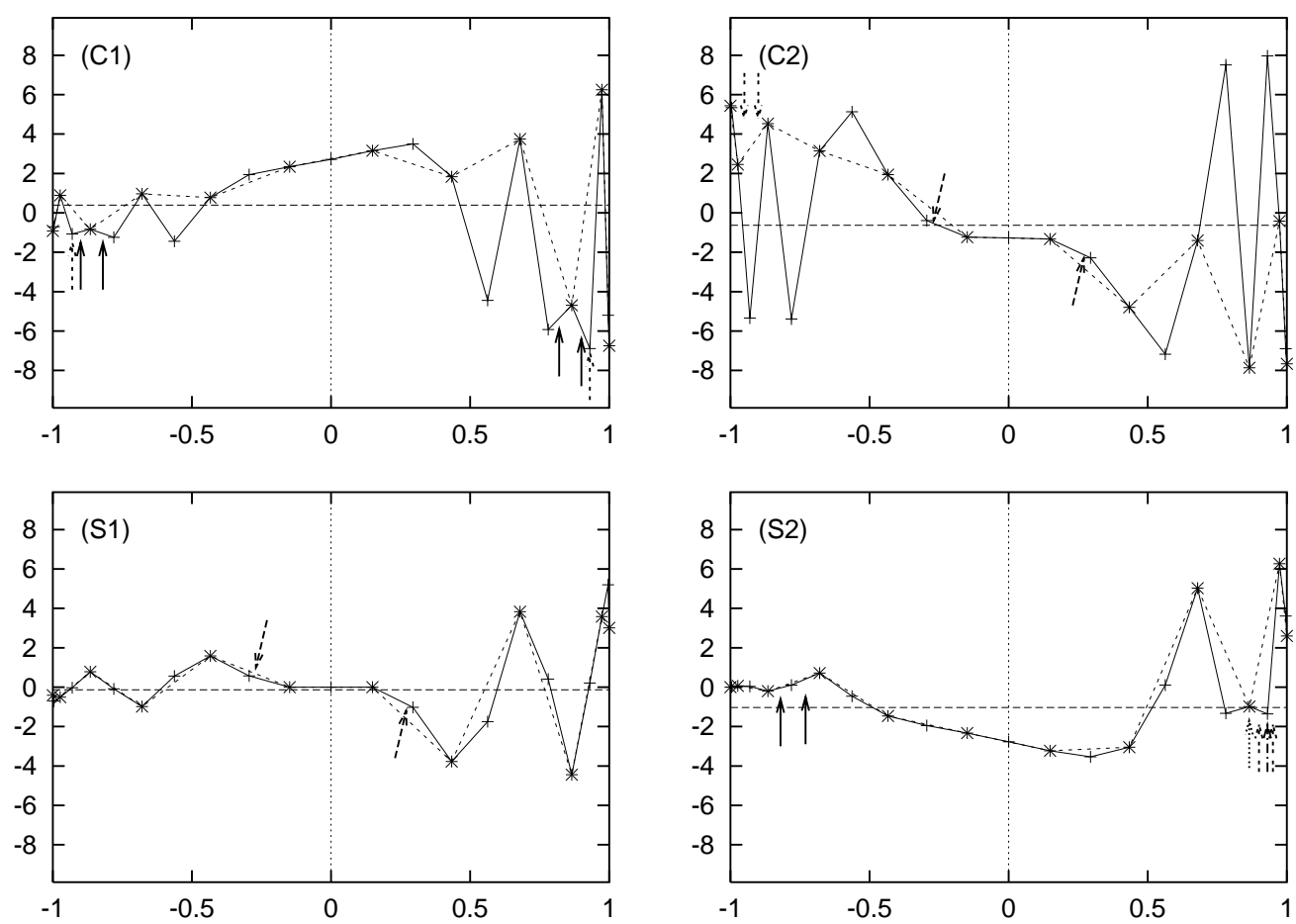

Fig. 7. Ill-conditioned integrand features in the family of integrals (25-29) at $\omega=1612 \pi / 60$.

Fig. 7 and fig. 8, show integrand profiles for the integrals (25-26) and (28$29)$ at the large parameter values $\omega=(1612 \pi / 60)$ and $\omega=(3646 \pi / 60)$ respectively, together with hints (showed by arrows) on infringements of the reliability criteria established in Sec. 4.

A scrutiny of the integrand profiles shows that, in general, it is hardly probable that a highly oscillatory integrand structure can be resolved at the existing quadrature knots. However, if intermediate unresolved structures are present, these induce, as a rule, infringements of one or more reliability criteria. The complete list of criteria infringements is given below: 

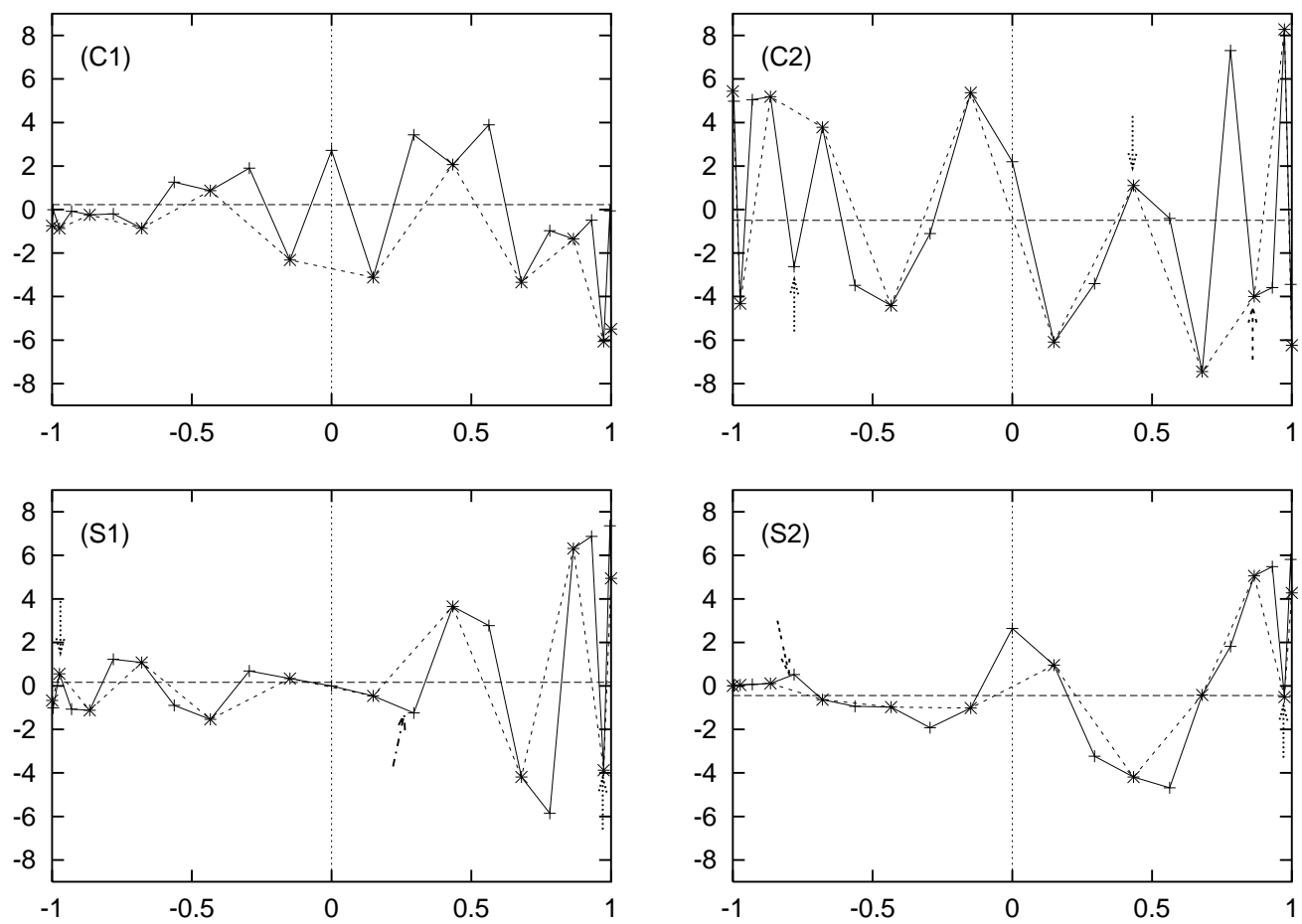

Fig. 8. Ill-conditioned integrand features in the family of integrals (25-29) at $\omega=3646 \pi / 60$.

- Criterion (I):

In fig 7: the integral (C1) at $y_{-8}$ and $y_{8}$; the integral (S2) at $y_{8}$.

In fig 8: the integral (S1) at $y_{-10}$ and $y_{10}$ (not shown in the plot); the integral (C2) at $y_{7}$, and the integral (S2) at $y_{10}$ (not shown in the plot).

- Criterion (IIa) - over end subranges:

In fig 8: the integral (S1) over the subrange $\left[y_{-11}, y_{-10}\right]$ (not shown in the plot).

- Criterion (IIa) - over inner subranges:

In fig 7: the integral $(\mathbf{C} 1)$ over the subranges $\left[y_{-8}, y_{-7}\right],\left[y_{-7}, y_{-6}\right],\left[y_{6}, y_{7}\right]$, and $\left[y_{7}, y_{8}\right]$; the integral (S2) over the subranges $\left[y_{-7}, y_{-6}\right]$ and $\left[y_{-6}, y_{-5}\right]$;

In fig 8: the integral (C1) over 15 (!) subranges: $\left[y_{-10}, y_{-9}\right],\left[y_{-9}, y_{-8}\right]$, $\left[y_{-8}, y_{-7}\right],\left[y_{-7}, y_{-6}\right],\left[y_{-6}, y_{-5}\right],\left[y_{-4}, y_{-3}\right],\left[y_{-3}, y_{-2}\right],\left[y_{-1}, y_{0}\right],\left[y_{2}, y_{3}\right] ;\left[y_{3}, y_{4}\right]$, $\left[y_{5}, y_{6}\right],\left[y_{6}, y_{7}\right],\left[y_{7}, y_{8}\right],\left[y_{8}, y_{9}\right]$, and $\left[y_{9}, y_{10}\right]$ (not shown in the plot since they are obvious).

- Criterion (IIb):

In fig 7: the integral (S2) at $y_{7}$;

In fig 8: the integral (S1) at $y_{-9}$ and $y_{9}$; the integral $(\mathbf{C 2})$ at $y_{-6}$ and $y_{3}$; the integral (S2) at $y_{9}$.

- Criterion (III):

In fig 7: the integral (C2) inside the subranges: $\left[y_{-9}, y_{-8}\right]$, and $\left[y_{-8}, y_{-7}\right]$; the integral (S2) inside the subranges: $\left[y_{7}, y_{8}\right]$, and $\left[y_{8}, y_{9}\right]$; 
- Criterion (IV):

In fig 8: the integral (S1) at $y_{9}$ (left derivative).

- Criterion ( $V)$ :

In fig 8: the integral (S2) at $y_{-6}$ (left neighbourhood).

- Criterion (VI):

In fig 7: the integral (S1) to the right of the knot $y_{-2}$ and to the left of the knot $y_{2}$; the integral (C2) to the right of the knot $y_{-2}$ and to the left of the knot $y_{2}$.

\section{Comments and conclusions}

The present investigation started from the need to get reliable numerical solutions of difficult parametric integrals occurring in theoretical models devoted to the study of the mechanism of the high- $T_{c}$ superconductivity in cuprates [1]-[5] and in a theoretical model of nuclear fission [6]. An important prerequisite to be satisfied by the automatic quadrature algorithms needed for the evaluation of the observables was the substantial increase of the reliability of the local error estimates.

We have found that the study of the conditioning of the integrand profile enables the formulation of validation criteria (consistency conditions for a well-conditioned profile) able to identify insufficient profile resolution or the occurrence of isolated difficult points of the integrand. The analysis is simple, it is intuitive, it is easily implemented in a computer program and it is easily done.

An important supplementary bonus offered by this analysis was the identification of $q_{2 n}$ output reliability ranges which are substantially larger in comparison with those obtained within the usual implementations of quadrature routines. The unsatisfactory features noticed in the validation criteria developed in ref. [8] have been fully removed.

The subroutines doing the profile analysis described in this paper are documented and described in a separate document [11].

We conclude this study with the observation that the validation analysis described in the present paper is not intended to replace the existing quadrature algorithms. When the estimated accuracy exceeds a critical threshold (tentatively set to five decimal figures), then the present procedure is skipped altogether. However, if this threshold is not attained, it is automatically activated by the general control routine. Its results prove to be invaluable in the analysis of complex integrands, where it is able to discover the overwhelming fraction of peculiar integrand profiles at early stages of the analysis. 


\section{Acknowledgments}

The investigation was partially financed by the JINR grant no. 571/15.10.2001 afforded by the Romanian Plenipotentiary Representative.

One author (Gh.A.) is grateful to Yu Lu, A. Săndulescu, and Ş. Mişicu for discussions of specific physical models.

\section{References}

[1] N.M. Plakida, R. Hayn, and J.-L. Richard, Phys. Rev. B 51 (1995) 16599.

[2] N.M. Plakida, L. Anton, S. Adam, and Gh. Adam, In New Trends in Superconductivity. J.F. Annett and S. Kruchinin, Eds. (Kluwer Academic Publ., New York, 2002) p. 29.

[3] N.M. Plakida, L. Anton, S. Adam, and Gh. Adam, Preprint JINR, E-17-200159, Dubna, 2001; arXiv:cond-mat/0104234.

[4] P.A. Marchetti, Zhao-Bin Su, and Lu Yu, Phys. Rev. B 58 (1998) 5808.

[5] P.A. Marchetti, Jian-Hui Dai, Zhao-Bin Su, and Lu Yu, J. Phys.: Cond. Matter 12 (2000) L329.

[6] A. Săndulescu, F. Cârstoiu, Ş. Mişicu, A. Florescu, A.V. Ramayya, J.H. Hamilton, J.K. Hwang, W. Greiner, Phys. Rev. C 57 (1998) 2321.

[7] A.R. Krommer and C.W. Ueberhuber. Computational Integration (SIAM, Philadelphia, 1998).

[8] Gh. Adam, S. Adam, Computer Phys. Commun. 135 (2001) 261.

[9] R. Piessens, E. deDoncker-Kapenga, C.W. Überhuber, and D.K. Kahaner, QUADPACK, a subroutine package for automatic integration (Springer Verlag, Berlin, 1983).

[10] A.S. Kronrod, Nodes and weights of quadrature formulas (Consultants Bureau, New York, 1965).

[11] Gh. Adam, S. Adam, QGKPAN - Quadrature at Gauss-Kronrod Knots with Profile Analysis, to be submitted to Computer Phys. Commun. 